\title{
Naturaleza de la ciencia: efecto de variables profesionales y sociodemográficas
}

Nature of Science: Effect of Professional and Sociodemographic Variables Nature de la science: l'effet de variables professionnelles et sociodémographiques

Natureza da ciência: efeitos de variáveis profissionais e sócio demográficas

Fecha de recepción: 26 DE JUNIO DE 2015/Fecha de aceptación: 20 DE FEBRERO DE 2017/Fecha de disponibilidad en línea: 1 DE DICIEMBRE DE 2017

Encuentre este artículo en http://magisinvestigacioneducacion.javeriana.edu.co/

Escrito por SILVIA TECPAN-FLoRES Departamento de Física, Universidad de Santiago de Chile Santiago, Chile silvia.tecpan@usach.cl, silviat.flores@itesm.mx

Genaro Zavala-EnríQuez Escuela de Ingeniería y Ciencias, Tecnológico de Monterrey MonterRey, México Facultad de Ingeniería, Universidad Andrés Bello, Santiago, Chile genaro.zavala@itesm.mx

\begin{abstract}
Resumen
Se realizó un estudio relacional cuantitativo con profesores de secundaria en México que cursaron un diplomado en competencia científica para diagnosticar las concepciones de naturaleza de la ciencia y su relación con variables sociodemográficas y de estatus profesional. Se utilizó una versión electrónica del Cuestionario de Opiniones sobre Ciencia, Tecnología y Sociedad (Manassero-Mas, Vázquez-Alonso \& Acevedo-Díaz, 2001). Los resultados concuerdan con investigaciones previas, cuyas concepciones de naturaleza de la ciencia de los profesores son principalmente "ingenuas" (Garritz, Rueda \& Robles, 2011). Las variables sociodemográficas mostraron mayor influencia en las concepciones de naturaleza de la ciencia que aquellas de estatus profesional. Se discuten las limitaciones del estudio.
\end{abstract}

\section{Palabras clave}

Ciencia y sociedad; enseñanza secundaria;

formación de docentes de secundaria; cultura científica; cuestionario COCTS

Para citar este artículo / To cite this article / Pour citer cet article / Para citar este artigo

Tecpan, Silvia \& Zavala, Genaro (2017). Naturaleza de la ciencia: efecto de variables profesionales y sociodemográficas. magis, Revista Internacional de Investigación en Educación, 10 (20), 11-34. doi: 10.11144/Javeriana.m10-20.ncev 


\section{Keywords}

Science and society; Secondary education;

teacher development of teachers

from high school; scientific culture;

COCTS questionnaire

\section{Mots clés}

Science et société; enseignement secondaire; formation des enseignants d'éducation secondaire; culture scientifique; questionnaire COCTS

\section{Abstract}

A quantitative relational study is presented in this article. This study was conducted with high school teachers in Mexico, who studied a diploma in scientific competence, in order to diagnose the conceptions of the nature of science and its relationship with sociodemographic variables and professional status. We used an electronic version of the Science, Technology and Society Opinions Questionnaire (Manassero, Vázquez \& Acevedo, 2001). Results agree with previous research studies: teachers' conceptions of nature of science are mainly "naive" (Garritz, Rueda \& Robles, 2011). Sociodemographic variables showed a greater influence in the conceptions of the nature of science than in those regarding professional status. Limitations of the study are discussed.

\section{Résumé}

Dans cet article de recherche on présente une étude relationnelle quantitative avec les enseignants d'éducation secondaire au Mexique qui ont accompli une spécialisation en compétence scientifique pour faire le diagnostic des conceptions de la Nature et de la Science et le rapport avec les variables sociodémographiques et de statut professionnel. On a utilisé une version électronique de Questionnaire d'avis par rapport à la Science, la Technologie et la Société (Manassero, Vázquez \& Acevedo, 2001). Les résultats coïncident avec les recherches préalables où les conceptions de nature de la science des enseignants sont principalement naïves (Garritz, Rueda \& Robles, 2011). Les variables sociodémographiques ont montrées une plus grande influence dans les conceptions de nature de la science que celles de statut professionnel. On discute par rapport aux contraintes de l'étude.

\section{Palavras-chave}

Ciência e sociedade; ensino secundário; formação de docentes de secundária; cultura científica; questionário COCTS

\section{Resumo}

Neste artigo de pesquisa apresenta-se um estudo relacional quantitativo com professores de secundária no México que cursaram um diplomado em competência científica para diagnosticar as concepções de natureza da ciência e sua relação com variáveis sócio demográficas e de status profissional. Utilizou-se uma versão eletrônica do questionário de opiniões sobre ciência, tecnologia e sociedade (Manassero, Vázquez \& Acevedo, 2001). Os resultados coincidem com pesquisas anteriores onde as concepções de natureza da ciência dos professores são principalmente "ingênuas" (Garritz, Rueda \& Robles, 2011). As variáveis sócio demográficas mostraram maior influência nas concepções de natureza da ciência que aquelas de status profissional. Discutem-se as limitações do estudo. 


\section{Introducción}

La competencia científica tal como ha sido establecida en el marco del Programa para la Evaluación Internacional de Alumnos (Programme for International Student Assessment, PISA) de la Organización para la Cooperación y el Desarrollo Económicos (OCDE) contempla la diferencia entre el conocimiento de las ciencias y el conocimiento sobre las ciencias (OCDE, 2008). No basta el conocimiento científico para enseñar ciencias, es necesario saber cómo se gesta este conocimiento. La naturaleza de la ciencia (en adelante, $\mathrm{NdC}$ ) es necesaria para comprender qué es la ciencia y cómo se lleva a cabo la investigación científica, recordando que es una actividad humana influenciada por la sociedad (Cañas, Martín-Díaz \& Nieda, 2007).

El conocimiento de la $\mathrm{NdC}$ se ha convertido en un asunto clave en la reforma educativa de diversos países (Lin, Lieu, Chen, Huang \& Chang, 2012). La educación científica ha transitado desde una perspectiva propedéutica hacia la alfabetización en ciencia y tecnología para todos (Mosquera-Suárez, 2011; Vázquez-Alonso, Acevedo-Díaz \& ManasseroMas, 2005), de la cual la adquisición de opiniones apropiadas e informadas acerca de qué son y cómo funcionan la ciencia y la tecnología y su relación con la sociedad es un componente esencial (Bennássar-Roig, Vázquez-Alonso, Manassero-Mas \& García-Carmona, 2011). El abandono del conductismo en la educación y el positivismo en la concepción de la ciencia también han influido para que la NdC sea cada vez más relevante en la práctica docente (López y Mota \& León-Trueba, 2003).

De forma reiterada se ha mencionado que una mejor comprensión de la NdC afecta positivamente la enseñanza de las ciencias (Flores-Camacho, 2012). Es necesario que los profesores sean capaces de ayudar a sus estudiantes a construir una visión actualizada de la NdC acorde con los planteamientos de la nueva filosofía de la ciencia (de Pro Bueno, 2003). Sin embargo, hallazgos de los últimos 20 años indican que los maestros de ciencias no poseen una adecuada concepción de $\mathrm{NdC}$, independientemente del instrumento utilizado para medirla (Lederman, 2007). Se sabe además que gran cantidad de profesores desconocen los avances en la didáctica de las ciencias y realizan su labor guiados por el sentido común (Talanquer, 2000).

En México, las ciencias ocupan un lugar secundario en la educación básica al considerar que es un conocimiento no esencial y poco útil para la vida cotidiana, por lo que de forma sistemática se han dedicado menores recursos al desarrollo de material didáctico y a la formación docente en ciencias (Flores-Camacho, 2012). No obstante, en la Reforma Educativa a la Educación Secundaria de 2006, la comprensión de NdC se incluye como un elemento central, para las asignaturas de ciencias (Candela, 2006). Este aspecto no ha sido incorporado plenamente en la formación docente (Flores-Camacho, Gallegos-Cázares \& Reyes-Cárdenas, 2007). Los docentes desempeñan un rol fundamental en los resultados que se obtienen en cualquier reforma educativa, pues deben orientar sus competencias y actitudes para modificar sus prácticas de acuerdo con estas reformas (Valdés-Cuervo, Arreola-Olivarría, Angulo-Armenta, Carlos-Martínez \& García-López, 2011), por lo que resulta relevante brindar la formación adecuada en $\mathrm{NdC}$.

A continuación, se presenta una breve revisión de investigaciones sobre las concepciones de $\mathrm{NdC}$ de profesores. Cabe mencionar que la revisión no es exhaustiva y abarca distintos niveles educativos y países.
Descripción del artículo | Article description | Description de l'article Artigo descrição

Este artículo de investigación forma parte del proyecto Determinación del impacto de características docentes que influyen en el diseño de secuencias didácticas para desarrollar la competencia científica. Los investigadores Silvia Tecpan y Genaro Zavala evalúan y describen las características de los docentes de secundaria relacionadas con la competencia científica. La investigación surge de la necesidad de identificar características de los profesores de ciencias en educación media básica mejor tipificadas que permitan avanzar en la formación y actualización de profesores a partir de evidencia. 


\section{Fundamentación teórica}

En México, los estudios sobre el docente de ciencias naturales de educación básica han sido mínimos (López y Mota \& León-Trueba, 2003). Los bajos resultados obtenidos en PISA 2000 alertaron sobre el endeble conocimiento disciplinar de los docentes de ciencias en secundaria (FloresCamacho, 2012), y se hizo evidente la necesidad de investigar las correlaciones entre las características del profesor de ciencias y el éxito en la enseñanza como parte de los estudios relacionados con la formación y actualización de profesores de ciencias.

Debido a que la $\mathrm{NdC}$ es un aspecto central en el aprendizaje de las ciencias, se ha establecido su relación con distintas variables. El principal aspecto que se ha investigado, desde 1990, es la relación entre la concepción de $\mathrm{NdC}$, la concepción de aprendizaje y la de enseñanza (Lederman, 2007). Aún no hay acuerdo, algunas investigaciones concuerdan en que estas concepciones determinan la práctica docente (Flores-Camacho, López-Mota, Gallegos-Cázares \& Barojas, 2000; Tsai, 2002), pero otras indican que hay un bajo nivel de conciencia sobre esta relación pues los docentes no reflexionan al respecto, por lo que no se puede establecer una relación directa entre la comprensión de la $\mathrm{NdC}$ y las prácticas educativas en el salón de clase (Barona-Ríos, Verjovsky, Moreno-Ruiz \& Lessard, 2004; Carvajal-Cantillo \& Gómez-Vallarta, 2002).

Otra variable relacionada con $\mathrm{NdC}$ es la experiencia como docente de ciencias. Se ha observado que no contribuye por sí misma a mejorar la comprensión de $\mathrm{NdC}$, pues no se promueve la reflexión crítica (Dogan \& Abd-El-Khalick, 2008).

La formación profesional inicial también se ha analizado. La comprensión de $\mathrm{NdC}$ entre profesores en formación que inician la universidad y los que finalizan sus estudios es similar, por lo que no hay evidencia empírica para sostener que los estudios aumenten la comprensión de NdC (Callejas-Restrepo \& Mendoza-Parada, 2011). Diversas investigaciones han encontrado que la formación profesional del docente de ciencias no tiene impacto en sus concepciones de NdC (Acevedo-Díaz, Vázquez-Alonso, Acevedo-Romero \& Manassero-Mas, 2002; Acevedo-Díaz, Vázquez-Alonso, Manassero-Mas \& Acevedo-Romero, 2002; Flores-Camacho, GallegosCázares \& Reyes-Cárdenas, 2007; Vázquez-Alonso, Manassero-Mas \& Talavera, 2010). Independientemente de los instrumentos empleados, se ha observado que domina la visión positivista de la ciencia (Flores-Camacho, Gallegos-Cázares \& Reyes-Cárdenas, 2007; Flores-Camacho, López-Mota, Gallegos-Cázares \& Barojas, 2000; López y Mota, Rodríguez-Pineda \& Bonilla-Pedroza, 2004; Verjovsky \& Waldegg, 2005; Tsai, 2002), y la enseñanza como transmisión de conocimientos (Tsai, 2002; Verjovsky \& Waldegg, 2005). Hay evidencia de que el conocimiento sobre $\mathrm{NdC}$ de los docentes de secundaria en México proviene principalmente de ambientes informales de aprendizaje, y no de su formación profesional, lo que contribuye a que tengan confusión para distinguir el conocimiento científico del que no lo es (Flores-Camacho, Gallegos-Cázares \& Reyes-Cárdenas, 2007).

La formación continua es otra variable que se ha analizado. Se ha encontrado que los cursos de actualización profesional han provocado cambios en el discurso referido a la $\mathrm{NdC}$ y el aprendizaje, pero no se observan en la práctica docente (López y Mota, Rodríguez-Pineda \& Bonilla-Pedroza, 2004; Verjovsky \& Waldegg, 2005).

Otras variables relacionadas con $\mathrm{NdC}$ han sido el grado académico del profesor y la región geográfica de procedencia. Ambas mostraron relación con el conocimiento de NdC en Turquía (Dogan \& Abd-El-Khalick, 2008), 
pero no se ha verificado en México. El conocimiento disciplinar tiene bajo impacto en el conocimiento de la $\mathrm{NdC}$, este último está relacionado estrechamente con la alfabetización científica (Chin, 2005). Por otra parte, se puede establecer que los profesores sostienen ideas previas sobre la didáctica de la ciencia de manera equivalente a las que sostienen los estudiantes al aprender ciencias y dificultan su aprendizaje de esta área de conocimiento (Mosquera-Suárez, 2011).

De acuerdo con Ángel Daniel López y Mota, Diana Patricia Rodríguez-Pineda y María Xóchitl BonillaPedroza (2004), se espera que los profesores de ciencias transiten de la visión dogmática, acumulativa y de verdades absolutas de la ciencia a una visión contemporánea que tome en cuenta el carácter dinámico de esta actividad humana en la que teorías y conceptos pueden cambiar con el tiempo. Es decir, se espera que transiten de una visión ingenua de la $\mathrm{NdC}$ basada en verdades absolutas, a una visión informada que considere su naturaleza cambiante.

Al comparar las concepciones de NdC entre estudiantes y profesores de ciencias se ha encontrado que ambas se alejan de la visión informada o contemporánea (García-Ruiz, Calixto-Flores \& Cid del Prado, 2011; Garritz, Rueda \& Robles, 2011).

\section{Establecimiento del problema}

La Organización de Naciones Unidas para la Educación, la Ciencia y la Cultura (UNESCO) reportó que México forma menos científicos que naciones con desarrollo similar como Brasil, Turquía y Corea del Sur y tiene menos producciones científicas y patentes (UNESCO, 2010), lo que sugiere bajo interés por la actividad científica. Por otra parte, en PISA 2006 y PISA 2009, México se situó entre los países con una diferencia estadísticamente significativa por debajo de la media de la OCDE, lo que manifiesta el bajo desarrollo de la competencia científica entre los jóvenes de 15 años que participaron en estos diagnósticos (OCDE, 2008: OECD, 2010). Este panorama muestra la urgencia de promover la competencia científica, uno de cuyos componentes esenciales es la comprensión de la NdC.

Para mejorar la competencia científica de los estudiantes es necesario que los docentes utilicen estrategias de enseñanza-aprendizaje de la ciencia específicas que permitan a sus alumnos aprender más y con mayor profundidad (Pozo-Municio \& Gómez-Crespo, 1998). Sin embargo, la falta de material didáctico que facilite a los profesores abordar estos contenidos y que, al mismo tiempo, contribuya a desarrollar el conocimiento pedagógico de contenido de la $\mathrm{NdC}$ puede ser una de las razones por las que los profesores no incluyen estos temas en sus secuencias didácticas (Lin, Lieu, Chen, Huang \& Chang, 2012).
De acuerdo con Yilmaz Cakici y Eylem Bayir (2012), los docentes de ciencias evitan abordar en sus clases aspectos esenciales de la NdC, pues ellos mismos sostienen concepciones ingenuas al respecto. Considerando la necesidad de incluir la $\mathrm{NdC}$ en las secuencias didácticas para promover la competencia científica y la relevancia de las concepciones docentes en la construcción de conocimiento relacionado con la $\mathrm{NdC}$, en esta investigación se buscó diagnosticar las concepciones de $\mathrm{NdC}$ de profesores de secundaria y su relación con otras variables demográficas y de estatus profesional.

\section{Objetivo general}

Diagnosticar las concepciones de $\mathrm{NdC}$ de los profesores de secundaria que cursan el diplomado en competencia científica por medio de un instrumento que permita obtener un índice normalizado del grado de coincidencia con las opiniones de los expertos y establecer su relación con variables sociodemográficas de los docentes y de estatus profesional como asignatura que imparten y formación profesional.

\section{Objetivos específicos}

a) Diagnosticar las concepciones de $\mathrm{NdC}$ de los profesores de secundaria que cursan el diplomado en competencia científica.

b) Establecer si hay relaciones significativas entre las concepciones de $\mathrm{NdC}$ y las variables sociodemográficas (género, edad, zona de procedencia) y de estatus profesional (formación profesional, asignatura que imparten).

\section{Metodología}

Se realizó un estudio cuantitativo transeccional relacional. La población estuvo compuesta por profesores de secundaria que cursaron el diplomado en desarrollo de la competencia científica, durante los ciclos escolares 2011-2012 y 2012-2013.

Muestra. No probabilística, integrada por 297 profesores de secundaria distribuidos a lo largo de toda la República mexicana. La muestra incluye docentes de telesecundaria. La participación fue voluntaria. Participaron 135 hombres y 162 mujeres.

Instrumento . Se utilizó el Cuestionario de Opiniones sobre Ciencia, Tecnología y Sociedad (COCTS, Manassero-Mas, Vázquez-Alonso \& Acevedo-Díaz, 2001). Los ítems tienen formato de selección múltiple, inician con una pregunta en la que se plantea un problema seguido por un conjunto de frases en escala Likert de nueve puntos con distintos grados de acuerdo. Una versión similar del instrumento empleado fue utilizada 
a gran escala en el Proyecto Iberoamericano de Evaluación de Actitudes Relacionadas con la Ciencia, la Tecnología y la Sociedad (PIEARCTS), en la publicación se incluyen las distintas versiones del instrumento en español y portugués validado por distintos grupos de investigación (BennássarRoig, García-Carmona, Vázquez-Alonso, Manassero-Mas \& Montesano de Talavera, 2011).

En este estudio se incluyeron 19 ítems con 131 frases distribuidas en 28 Adecuadas, que expresan un punto de vista coherente con los conocimientos de historia, epistemología y sociología de la ciencia; 57 Plausibles, que son parcialmente coherentes; y 46 Ingenuas, que son aquellas que expresan un punto de vista que no es ni adecuado ni plausible, de acuerdo con el manual de uso del instrumento (Manassero-Mas, Vázquez-Alonso \& Acevedo-Díaz, 2001). En la tabla 1 se muestra como ejemplo el ítem 90621 (de acuerdo con la nomenclatura establecida por los autores del instrumento) con la información que se presentó a los participantes.

Tabla 1

Ítem 90621 con la información que se presenta al participante

Situación problema y afirmaciones a valorar con escala Likert de nueve puntos

\begin{tabular}{|c|c|c|c|c|c|c|c|c|c|c|}
\hline \multicolumn{11}{|c|}{ 18. Los mejores científicos son los que siguen las etapas del n } \\
\hline \multicolumn{2}{|r|}{$\begin{array}{l}\text { Para cada una de las frases siguientes, marca el número de la escala } \\
\text { que represente mejor el grado de acuerdo entre tu propia opinión y la } \\
\text { posición expuesta en la frase. Donde } 1 \text { significa desacuerdo total y } 9, \\
\text { acuerdo total }\end{array}$} & \multicolumn{9}{|c|}{ Grado de acuerdo } \\
\hline A & $\begin{array}{l}\text { El método científico asegura resultados válidos, claros, lógicos y } \\
\text { exactos. Por tanto, la mayoría de los científicos seguirá las etapas } \\
\text { del método científico }\end{array}$ & 1 & 2 & 3 & 4 & 5 & 6 & 7 & 8 & 9 \\
\hline B & $\begin{array}{l}\text { El método científico, tal como se enseña en las clases, debería } \\
\text { funcionar bien para la mayoría de los científicos }\end{array}$ & 1 & 2 & 3 & 4 & 5 & 6 & 7 & 8 & 9 \\
\hline C & $\begin{array}{l}\text { El método científico es útil en muchos casos, pero no asegura } \\
\text { resultados. Por tanto, los mejores científicos también tendrán } \\
\text { originalidad y creatividad }\end{array}$ & 1 & 2 & 3 & 4 & 5 & 6 & 7 & 8 & 9 \\
\hline$D$ & $\begin{array}{l}\text { Los mejores científicos son aquellos que usan cualquier método } \\
\text { para obtener resultados favorables (incluyendo la imaginación y } \\
\text { la creatividad) }\end{array}$ & 1 & 2 & 3 & 4 & 5 & 6 & 7 & 8 & 9 \\
\hline$E$ & $\begin{array}{l}\text { Muchos descubrimientos científicos fueron hechos por } \\
\text { casualidad, y no siguiendo el método científico }\end{array}$ & 1 & 2 & 3 & 4 & 5 & 6 & 7 & 8 & 9 \\
\hline
\end{tabular}

Fuente: adaptado de María Antonia Manassero-Mas, Ángel Vázquez-Alonso y José Antonio Acevedo-Díaz (2001)

Cada frase está clasificada como Adecuada, Plausible o Ingenua. La clasificación fue determinada previamente por un grupo de 11 expertos (investigadores en didáctica de la ciencia, profesores de ciencia y filósofos) desde la perspectiva de los conocimientos de historia, epistemología, filosofía y sociología de la ciencia vigentes. Para mayor detalle del procedimiento seguido en la integración del comité de expertos y la clasificación de frases que integran el COCTS, se puede consultar Ángel VázquezAlonso, José Antonio Acevedo-Díaz y María Antonia Manassero-Mas (2005), quienes dan cuenta del proceso y detallan los análisis estadísticos (análisis de conglomerados y discriminante) que confirman la clasificación.

Los participantes no tienen conocimiento de la clasificación de las frases al momento de valorar las afirmaciones. Cuando se establece un grado de acuerdo alto en una frase clasificada como Adecuada se considera 
que hay concordancia con los expertos, por el contrario cuando se tiene un grado de acuerdo alto en una frase clasificada como Ingenua se considera que la opinión es opuesta a la de los expertos. Las puntuaciones directas que corresponden al grado de acuerdo que establece el participante tienen valor distinto dependiendo de la clasificación a que corresponda cada frase (Vázquez-Alonso, Acevedo-Díaz, Manassero-Mas \& AcevedoRomero, 2006), siguiendo las puntuaciones que se indican en la tabla 2.

Tabla 2

Significado de las puntuaciones directas de acuerdo/desacuerdo según la clasificación de cada frase

\begin{tabular}{l|c|c|c|c|c|c|c|c|c}
\hline \multirow{2}{*}{ Escala directa } & \multicolumn{7}{|c}{ Transformación de las puntuaciones directas } \\
\cline { 2 - 11 } & 1 & 2 & 3 & 4 & 5 & 6 & 7 & 8 & 9 \\
\hline Adecuadas & -4 & -3 & -2 & -1 & 0 & 1 & 2 & 3 & 4 \\
\hline Plausibles & -2 & -1 & 0 & 1 & 2 & 1 & 0 & -1 & -2 \\
\hline Ingenuas & 4 & 3 & 2 & 1 & 0 & -1 & -2 & -3 & -4 \\
\hline \hline
\end{tabular}

Fuente: adaptado de Ángel Vázquez-Alonso, José Antonio Acevedo-Díaz, María Antonia Manassero-Mas y Pilar Acevedo-Romero (2006)

En la tabla 3 se ejemplifica la selección directa que realizó un participante (en negritas y subrayado), la categoría a la cual corresponde cada frase y finalmente la conversión que permite calcular el índice por categoría para esa persona, en ese ítem, el cual cuenta con dos frases Plausibles (P), dos frases Ingenuas (I) y una Adecuada (A).

Tabla 3

Ítem 90621 con valoración directa de un participante (en negrita y subrayado), clasificación de frases y conversión en función de la categoría y el grado de acuerdo

\begin{tabular}{|c|c|c|c|c|c|c|c|c|c|c|c|c|}
\hline \multirow{2}{*}{\multicolumn{2}{|c|}{$\begin{array}{l}\text { 18(90621). Los mejores científicos son los que siguen las e } \\
\text { Para cada una de las frases siguientes, marca el número de la escala } \\
\text { que represente mejor el grado de acuerdo entre tu propia opinión } \\
\text { y la posición expuesta en la frase }\end{array}$}} & \multicolumn{8}{|c|}{ Grado de acuerdo } & & & \\
\hline & & \multicolumn{3}{|c|}{ Bajo } & \multicolumn{3}{|c|}{ Medio } & \multicolumn{3}{|c|}{ Alto } & 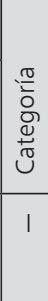 & 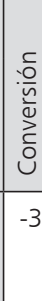 \\
\hline B & $\begin{array}{l}\text { El método científico, tal como se enseña en las clases, debería } \\
\text { funcionar bien para la mayoría de los científicos }\end{array}$ & 1 & 2 & 3 & 4 & 5 & 6 & 7 & 8 & $\underline{9}$ & । & -4 \\
\hline $\mathrm{D}$ & $\begin{array}{l}\text { Los mejores científicos son aquellos que usan cualquier método } \\
\text { para obtener resultados favorables (incluyendo la imaginación y } \\
\text { la creatividad) }\end{array}$ & 1 & 2 & 3 & 4 & 5 & 6 & 7 & 8 & $\underline{9}$ & $P$ & -2 \\
\hline$E$ & $\begin{array}{l}\text { Muchos descubrimientos científicos fueron hechos por } \\
\text { casualidad, y no siguiendo el método científico }\end{array}$ & 1 & 2 & 3 & $\underline{4}$ & 5 & 6 & 7 & 8 & 9 & $P$ & 1 \\
\hline
\end{tabular}

Fuente: adaptado de María Antonia Manassero-Mas, Ángel Vázquez-Alonso y José Antonio Acevedo-Díaz (2001),

Ángel Vázquez-Alonso, José Antonio Acevedo-Díaz, María Antonia Manassero-Mas y Pilar Acevedo-Romero (2006) 
Para obtener el índice por categoría se suma la puntuación convertida de las frases de la categoría, en el ejemplo, para las frases Ingenuas se tendría $(-3+-4)$ y ese resultado se divide entre el producto del número de frases de la categoría presentes en el ítem, en este caso 2, por la puntuación más alta positiva de la categoría, que de acuerdo con la tabla 2 para las frases Ingenuas es $4(2 \times 4)$. Para el ítem que se ejemplifica en la tabla 3, los índices por categoría son los siguientes:

$$
\begin{aligned}
& \text { Índice de frases Ingenuas }=\frac{\sum f l}{4 n_{l}}=\frac{-7}{8}=-.875 \\
& \text { Índice de frases Adecuadas }=\frac{\sum f A}{4 n_{A}}=\frac{3}{4}=.750 \\
& \text { Índice de frases Plausibles }=\frac{\sum f P}{4 n_{P}}=\frac{-1}{8}=-.125
\end{aligned}
$$

El índice global ponderado se obtiene al promediar los índices por categoría, de modo que el índice global ponderado para el ejemplo que se analiza es:

$$
\text { Índice ponderado }=\frac{I_{A}+I_{P}+I_{I}}{3}=-.08
$$

Los 19 ítems del instrumento empleado están organizados en seis dimensiones. De acuerdo con Heidi Celina Oviedo-Acevedo y Adalberto Campo-Arias (2005), se evaluó la consistencia interna de cada una de las dimensiones por medio del alfa de Cronbach. En este caso, todas superan el valor de .70 considerado aceptable (Vogt, 2007), con excepción de la dimensión Características de los científicos, que presenta una consistencia interna baja; sin embargo, se ubica en un valor aceptable (Oviedo-Acevedo \& Campo-Arias, 2005). En la tabla 4, se consignan el valor de alfa de Cronbach, el indicador de cada dimensión y los ítems que la integran.

Tabla 4

Dimensiones, confiabilidad, indicadores e ítems del instrumento empleado

\begin{tabular}{l|c|l|l}
\hline Dimensión & Alfa de Cronbach & Indicador & Ítems \\
\hline Ciencia y tecnología & .843 & Grado de acuerdo con la definición de ciencia y tecnología. & $10111,10113,10211$ \\
\hline $\begin{array}{l}\text { Influencia de la } \\
\text { sociedad sobre la } \\
\text { ciencia/tecnología }\end{array}$ & .722 & $\begin{array}{l}\text { Grado de acuerdo de la influencia de la sociedad } \\
\text { sobre instituciones educativas, sobre los científicos } \\
\text { y en general. }\end{array}$ & $\begin{array}{l}20511,20711, \\
20821\end{array}$ \\
\hline $\begin{array}{l}\text { Influencia de } \\
\text { ciencia/tecnología } \\
\text { sobre la sociedad }\end{array}$ & .730 & $\begin{array}{l}\text { Grado de acuerdo con la influencia de la ciencia y la } \\
\text { tecnología en la responsabilidad social, el bienestar } \\
\text { económico y en general. }\end{array}$ & $\begin{array}{l}40111,40511, \\
40821\end{array}$ \\
\hline $\begin{array}{l}\text { Influencia de la } \\
\text { ciencia escolar } \\
\text { sobre la sociedad }\end{array}$ & .760 & $\begin{array}{l}\text { Grado de acuerdo con la influencia de la ciencia escolar } \\
\text { en la unión de dos culturas y el fortalecimiento social. }\end{array}$ & 50111,50211 \\
\hline $\begin{array}{l}\text { Características de } \\
\text { los científicos }\end{array}$ & .621 & $\begin{array}{l}\text { Grado de acuerdo con las características de los científicos } \\
\text { como motivaciones, capacidades y efectos de género. }\end{array}$ & $\begin{array}{l}60111,60411, \\
60511\end{array}$ \\
\hline $\begin{array}{l}\text { Naturaleza del } \\
\text { conocimiento } \\
\text { científico }\end{array}$ & .858 & $\begin{array}{l}\text { Grado de acuerdo con la naturaleza del conocimiento } \\
\text { científico en los modelos científicos, provisionalidad del } \\
\text { conocimiento, aproximación a las investigaciones, y el } \\
\text { estatus epistemológico. }\end{array}$ & $\begin{array}{l}90211,90411, \\
90611,90621, \\
91011\end{array}$ \\
\hline \hline
\end{tabular}

Fuente: adaptado de María Antonia Manassero-Mas, Ángel Vázquez-Alonso y José Antonio Acevedo-Díaz (2001) 
Las dimensiones del instrumento concuerdan con la taxonomía de objetos actitudinales relacionados con temas de NdC propuesta por María Antonia Manassero y Ángel Vázquez-Alonso (2001), por lo que se considera que cuenta con validez de contenido. La validez de criterio no ha sido reportada y esto podría explicarse por la dificultad para establecer acuerdos sobre los temas de $\mathrm{NdC}$ que tienen naturaleza cambiante (Vázquez-Alonso, Manassero-Mas, AcevedoDíaz \& Acevedo-Romero, 2007).

\section{Procedimiento de recolección y análisis de datos}

Una vez que se contó con la autorización de la coordinación del diplomado, se colocó el instrumento en la plataforma del curso y se solicitó la participación voluntaria a los profesores a quienes se les garantizó que sus respuestas serían confidenciales y sin ningún efecto en su calificación. Los datos fueron transformados en variables numéricas y se prepararon para su análisis con el software Statistical Program for the Social Sciences, SPSS ${ }^{\circledR}$ versión 20. Por medio de la prueba $t$ de Student, se comparó si dos grupos difieren entre sí de manera significativa respecto a sus medias. Cuando se compararon más de dos grupos se eligió análisis de varianza de un factor (ANOVA) para determinar la existencia de diferencias al comparar distintas variables sociodemográficas y profesionales. En todos los casos se verificaron los supuestos estadísticos necesarios para la validez de la prueba (Vogt, 2007). Se utilizó un nivel de significación de .05.

\section{Resultados}

En primer lugar se presenta el diagnóstico de las concepciones de NdC de los profesores y enseguida la relación de los índices del COCTS con otras variables del profesor.

\section{Diagnóstico de las concepciones de $N d C$}

Se calculó el índice global para cada tipo de frases (Adecuada, Plausible e Ingenua) y el índice global ponderado que se obtiene al promediar el índice de los tres tipos. En la tabla 5 se puede observar que la categoría Adecuada aporta un valor positivo, mientras que las categorías Plausible e Ingenua aportan en sentido negativo. El índice global ponderado toma valores de -1 a +1 . Los valores cercanos a +1 indican que las opiniones concuerdan con las de los expertos. Se observa un sesgo hacia valores negativos para toda la muestra, ya que el índice global ponderado es negativo y próximo a cero $(\bar{x}=-.024, D E=.103)$, lo que sugiere que las concepciones de $\mathrm{NdC}$ de los profesores se alejan de las que proponen los expertos.

Tabla 5

Parámetros estadísticos de la distribución de la media de los índices actitudinales normalizados $(-1,+1)$ correspondientes a las respuestas de la muestra respecto al conjunto de ítems del COCTS

\begin{tabular}{l|c|c|c|c}
\hline & Adecuadas $^{\mathbf{a}}$ & Plausibles $^{\mathbf{a}}$ & Ingenuas $^{\mathbf{a}}$ & Índice global ponderado $^{\mathbf{b}}$ \\
\hline Media & .508 & -.426 & -.153 & -.024 \\
\hline Desviación estándar, DE & .153 & .246 & .190 & .103 \\
\hline Máximo & .87 & .27 & .49 & .23 \\
\hline Mínimo & -.02 & -.99 & -.56 & -.41 \\
\hline Rango & .89 & 1.26 & 1.05 & .64 \\
\hline \hline
\end{tabular}

Fuente: elaboración propia

a. Promedio de las medias de los índices normalizados de las frases adecuadas, plausibles e ingenuas, respectivamente de cada estudiante

b. Promedio de las medias de los tres anteriores (adecuadas, plausibles, ingenuas) 
En la tabla 6 se muestran los índices de las frases Adecuadas, Plausibles, Ingenuas y el índice global ponderado para cada ítem que están ordenados de forma consecutiva, tal como se presentaron en el cuestionario en línea. Se observa que las frases Adecuadas aportaron valores positivos en la mayoría de los ítems (excepto el 90611), en las frases Ingenuas se obtuvieron más valores negativos que positivos y en las frases Plausibles todos los valores fueron negativos. Se utiliza la nomenclatura propuesta por los autores del instrumento para facilitar la comparación con otros estudios.

El ítem que obtuvo el promedio más alto fue el $60511(\bar{x}=.31$, $\mathrm{DE}=.158)$ de la dimensión Características de los científicos/efectos de género. Cabe mencionar que la confiabilidad de la dimensión donde se ubica este ítem es menor a .70, por lo que los resultados deben revisarse con reserva. Aunque el índice global ponderado es bajo, el índice de las frases Adecuadas es .83 cercano a la opinión de los expertos; esto sugiere que los profesores están conscientes del origen de las diferencias en los descubrimientos científicos, saben que no tienen nada qué ver con el género del investigador, sino con sus diferencias individuales, lo que se refuerza con el resultado de las frases Ingenuas con un valor de .73 también cercano a la opinión de los expertos. Sin embargo, las frases Plausibles tienen un valor muy bajo -.64, lo que revela que los profesores se alejan del criterio de los expertos en las frases parcialmente correctas.

En sentido opuesto, en la tabla 6 se observa que el ítem con mayor distanciamiento con respecto a la opinión de los expertos es el 90611 $(\bar{x}=-.411, D E=.321)$, que corresponde a la dimensión Naturaleza del conocimiento científico/aproximación a las investigaciones. Los índices de los tres tipos de frases son negativos y por consecuencia también lo es el índice global ponderado, lo que indica que los profesores sostienen creencias ingenuas, como definir el método científico como el mero registro de datos de manera cuidadosa o reducirlo al control de variables experimentales para obtener un resultado libre de interpretación.

El siguiente ítem con alta puntuación es el 20511 de la dimensión Influencia de la sociedad sobre ciencia y tecnología/instituciones educativas. La confiabilidad de la dimensión de este ítem es de .722, por lo que se sugiere cautela con los resultados. La coincidencia con los expertos en las frases Adecuadas es alta .72, lo que sugiere que los profesores están de acuerdo en que se deben fomentar más los cursos de ciencias que permitan a los alumnos entender cómo la ciencia y la tecnología afectan su vida diaria. Las frases Ingenuas tuvieron menos concordancia con un valor de .46, lo que muestra que los profesores no están del todo de acuerdo en que los alumnos estudien más ciencias. Nuevamente, las frases Plausibles se alejan de la opinión de los expertos con un valor bajo -.45, lo que lleva a un índice global ponderado bajo 24 .

El ítem 40511 de la dimensión Influencia de ciencia y tecnología sobre la sociedad/bienestar económico muestra un índice global ponderado bajo .23, pero es el tercero más alto. La confiabilidad de la dimensión de este ítem es de .730 , por lo que se sugiere cautela con los resultados. La coincidencia con las frases Adecuadas es de .67, por lo que se puede mencionar que los profesores están de acuerdo en que el mayor desarrollo de un país en ciencia y tecnología puede acompañarse de mayor bienestar económico; sin embargo, la coincidencia con los expertos en las frases Ingenuas no es tan alta .49, lo que sugiere que los profesores dudan que mayor inversión en ciencia y tecnología pueda ser un beneficio económico para un país. Las frases Plausibles aportaron un valor negativo -.48 al índice global ponderado. 
Tabla 6

Índice global ponderado y por tipo de frase para cada ítem en escala de -1 a +1

\begin{tabular}{|c|c|c|c|c|c|}
\hline $\begin{array}{l}\text { Ítem } \\
\text { (Pregunta) }\end{array}$ & Tema & $\begin{array}{l}\text { Frases } \\
\text { Adecuadas }\end{array}$ & $\begin{array}{l}\text { Frases } \\
\text { Plausibles }\end{array}$ & $\begin{array}{l}\text { Frases } \\
\text { Ingenuas }\end{array}$ & $\begin{array}{l}\text { Índice global } \\
\text { ponderado }\end{array}$ \\
\hline $10111(1)$ & Definición de ciencia & .63 & -.46 & .07 & .08 \\
\hline $10113(2)$ & Definición de ciencia & .76 & -.42 & -.53 & -.06 \\
\hline $10211(3)$ & Definición de tecnología & .56 & -.44 & -.78 & -.22 \\
\hline $20511(4)$ & Instituciones educativas & .72 & -.45 & .46 & .24 \\
\hline $20711(5)$ & Influencia/científicos & .69 & -.40 & .07 & .12 \\
\hline $20821(6)$ & Influencia general & .44 & -.39 & -.18 & -.04 \\
\hline $40111(7)$ & Responsabilidad social & .66 & -.22 & -.39 & .02 \\
\hline $40511(8)$ & Bienestar económico & .67 & -.48 & .49 & .23 \\
\hline $40821(9)$ & Influencia general & 0 & -.44 & .12 & -.16 \\
\hline $50111(10)$ & Unión de dos culturas & .75 & -.23 & .12 & .21 \\
\hline $50211(11)$ & Fortalecimiento social & .08 & -.36 & -.58 & -.29 \\
\hline $60111(12)$ & Motivación de científicos & .15 & -.43 & -.27 & -.19 \\
\hline 60411 (13) & Capacidades de científicos & .48 & -.49 & .37 & .12 \\
\hline $60511(14)$ & Efectos de género & .83 & -.64 & .73 & .31 \\
\hline $90211(15)$ & Modelos científicos & .29 & -.39 & -.30 & -.13 \\
\hline $90411(16)$ & Provisionalidad & .68 & -.29 & -.19 & .07 \\
\hline $90611(17)$ & Aproximación a investigaciones & -.33 & -.51 & -.40 & -.41 \\
\hline $90621(18)$ & Aproximación a investigaciones & .62 & -.37 & -.28 & -.01 \\
\hline 91011 (19) & Estatus epistemológico & .64 & -.27 & -.40 & -.01 \\
\hline
\end{tabular}

Fuente: elaboración propia

El segundo ítem con puntuación baja en el índice global ponderado es el 50211 de la dimensión Influencia de la ciencia escolar sobre la sociedad/fortalecimiento social con un valor de -.29. En este ítem, las frases Adecuadas aportan un valor positivo, pero muy bajo de .08, mientras que las frases Ingenuas muestran un valor negativo alto de -.58 , lo que sugiere que los profesores difieren de la opinión de los expertos y consideran que las clases de ciencias los vuelven mejores consumidores. Las frases Plausibles también aportaron un valor negativo -.36.

El tercer ítem con puntuación baja es el 10211 de la dimensión Definición de ciencia y tecnología con un índice global ponderado de -.22. En este ítem, las frases Adecuadas aportan un valor positivo de .56, lo que sugiere que los profesores tienen una concepción relativamente adecuada de la definición de tecnología; sin embargo, las frases Ingenuas aportan un valor negativo alto de -.78 , lo que indica que sus opiniones 
están en el extremo opuesto a las de los expertos por lo que se puede mencionar que los profesores mantienen la concepción de que la tecnología es ciencia aplicada.

Continuando con este análisis, se puede establecer que los profesores de la muestra exhiben en su mayoría concepciones ingenuas de $\mathrm{NdC}$, pues los índices de las frases Adecuadas se ven contrarrestados por los índices de las frases Ingenuas y Plausibles, de las que se encontraron valores negativos cercanos a - 1 , lo que indica que las opiniones de los profesores están al extremo opuesto de la opinión de los expertos. Los resultados indican que los profesores tendrán dificultades para desarrollar la competencia científica en sus alumnos por lo que es necesario mejorar la formación docente en estos aspectos.

\section{Relación entre los indices del COCTS y variables sociodemográficas}

Género. Para determinar si hay diferencias entre los índices globales y por ítems, al comparar por género se recurrió a la prueba $t$ de Student para muestras independientes que permite determinar si las medias de dos poblaciones distribuidas en forma normal son iguales. Para aplicar la prueba, se verificó la forma de la distribución y la homogeneidad de la varianza de ambas muestras (Vogt, 2007). Los resultados globales evidencian que no hay diferencia estadísticamente significativa con respecto al género en las concepciones de $\mathrm{NdC}$. Únicamente en el índice de las frases ingenuas del ítem 10111 Definición de ciencia, se encontraron diferencias $(p=.004$, $t=2.903, d f=295)$, los hombres mostraron una media mayor $(\bar{x}=.147$, $\mathrm{SD}=.389)$ que las mujeres $(\bar{x}=.011, \mathrm{SD}=.408)$, lo que sugiere mayor coincidencia con los expertos. Siguiendo a Manuel Cárdenas-Castro y Héctor Arancibia-Martini (2014), se utilizó el programa estadístico G*Power (Faul, Erdfelder, Lang \& Buchner, 2007) para calcular el tamaño del efecto de esta diferencia, la cual es pequeña $(d=.33)$, con potencia estadística adecuada (81\%).

Edad. Se establecieron tres grupos de profesores de acuerdo con su edad, siguiendo la teoría de la adultez emergente (Arnett, 2008). El primero denominado Joven de 22 a 35 años contó con 95 profesores, el segundo denominado Adulto joven de 36 a 45 años tuvo 103 integrantes y el último denominado Adulto maduro agrupó a 99 profesores mayores de 45 años. Para determinar si hay diferencias entre las medias de estos grupos se utilizó ANOVA de un factor, previa verificación de supuestos estadísticos (Sheskin, 2007). Para determinar el sentido de las diferencias, cuando estas ocurrieron, se aplicó una prueba post hoc con el método Bonferroni. Se encontraron diferencias estadísticamente significativas en el índice total de las frases Plausibles ( $p=.002, F=6.498$, df $=2,294$ ), los jóvenes mostraron mayor grado de acuerdo con los expertos. El tamaño del efecto de esta comparación fue pequeño $(f=.20)$, la potencia de la prueba fue de $89 \%$ que supera el valor de referencia de $80 \%$ (Faul, Erdfelder, Lang \& Buchner, 2007).

Al revisar el comportamiento de los índices para las frases de cada ítem, se encontró que la variable edad fue la que aportó mayor cantidad de diferencias en cuatro ítems y cinco índices, lo que sugiere que cada grupo etario sostiene distintas concepciones en esos temas. Los resultados de la prueba ANOVA se muestran en la tabla 7, en que se observa que en todos los casos el efecto de las diferencias encontradas es pequeño de acuerdo a los criterios propuestos por Franz Faul, Edgar Erdfelder, Albert-Georg Lang y Axel Buchner (2007). 
Tabla 7

Ítems con diferencia estadísticamente significativa en función de la edad en algún índice, parámetros de la prueba ANOVA, tamaño del efecto (f) y potencia estadística

\begin{tabular}{|c|c|c|c|c|c|c|c|}
\hline 90411 Provisionalidad & Plausibles & Joven & 7.739 & 2,294 & .001 & .22 & .93 \\
\hline \multirow[t]{2}{*}{91011 Estatus epistemológico } & Plausible & Joven & 5.779 & 2,294 & .003 & .19 & .85 \\
\hline & Ponderado & Joven & 6.675 & 2,294 & .001 & .20 & .89 \\
\hline
\end{tabular}

Fuente: elaboración propia. ${ }^{*} p \leq .05$

Región. Para evaluar si hay diferencias en las concepciones de $\mathrm{NdC}$ asociadas a la región de procedencia se propusieron cinco regiones, acordes con la clasificación propuesta por el Consejo Nacional de Ciencia y Tecnología (CONACYT). En la tabla 8 se muestra la distribución de profesores en regiones y el máximo grado de estudios.

Tabla 8

Zona de procedencia y grado de estudios de los profesores de la muestra

\begin{tabular}{|c|c|c|c|c|}
\hline Zona de procedencia & Posgrado & Normalista & Universitario & Total \\
\hline $\begin{array}{l}\text { Metropolitana: Estado de México, } \\
\text { Distrito Federal, Morelos }\end{array}$ & 20 & 19 & 39 & 78 \\
\hline $\begin{array}{l}\text { Centro: Aguascalientes, Querétaro, } \\
\text { Guanajuato, San Luis Potosí }\end{array}$ & 29 & 31 & 15 & 75 \\
\hline $\begin{array}{l}\text { Oriente-centro: Hidalgo, Puebla, } \\
\text { Tlaxcala y Sur: Guerrero, Oaxaca, Chiapas }\end{array}$ & 25 & 10 & 32 & 67 \\
\hline $\begin{array}{l}\text { Occidente: Jalisco, Nayarit, Colima y } \\
\text { Michoacán }\end{array}$ & 12 & 20 & 19 & 51 \\
\hline $\begin{array}{l}\text { Norte: Chihuahua, Durango, Zacatecas, } \\
\text { Baja California Norte, Baja California Sur, } \\
\text { Sonora y Sinaloa, Nuevo León, Coahuila, } \\
\text { Tamaulipas }\end{array}$ & 9 & 5 & 12 & 26 \\
\hline Totales & 95 & 85 & 117 & 297 \\
\hline
\end{tabular}

Fuente: elaboración propia

La diferencia entre las concepciones de los profesores de cada región se verificó con ANOVA de un factor. Se encontraron diferencias estadísticamente significativas en el índice total de las frases Ingenuas ( $p=.006$, $\mathrm{F}=3.683, \mathrm{df}=4,292)$, el efecto fue pequeño $(f=.21)$ con una potencia estadística aceptable (86\%); y en el índice ponderado total ( $p=.011$, $\mathrm{F}=3.354, \mathrm{df}=4,292)$ donde el efecto es pequeño $(f=.20)$ y la potencia estadística aceptable (82\%). Los índices de cuatro ítems también mostraron diferencias que se verificaron con una prueba post hoc con el método Bonferroni. Los resultados se aprecian en la tabla 9. 
Tabla 9

Ítems con diferencia estadísticamente significativa en función de la región en algún índice, parámetros de la prueba ANOVA, tamaño del efecto (f) y potencia estadística

\begin{tabular}{l|l|l|l|l|l|l|l}
\hline Ítem & $\begin{array}{l}\text { Tipo de } \\
\text { índice }\end{array}$ & $\begin{array}{l}\text { Grupo con } \\
\text { mayor promedio }\end{array}$ & F & Df & p & $\begin{array}{l}\text { Potencia } \\
\text { estadística }\end{array}$ \\
\hline 20711 Influencia/científicos & Ingenuas & Norte & 3.509 & 4,292 & .008 & .21 & .84 \\
\hline 40821 Influencia general de CyT & Ingenua & Norte & 3.273 & 4,292 & .012 & .20 & .81 \\
\hline 90211 Modelos científicos & Ponderado & Norte & 3.132 & 4,292 & .015 & .20 & .82 \\
\hline 90411 Provisionalidad & Adecuadas & Metropolitana & 3.262 & 4,292 & .012 & .20 & .82 \\
\hline \hline
\end{tabular}

Fuente: elaboración propia. ${ }^{*} p \leq .05$

\section{Relación entre los indices del COCTS y las variables de estatus profesional}

Nivel de estudios. Se clasificó a los profesores en tres grupos, como se observó en la tabla 8. La diferencia entre las concepciones de NdC de estos grupos se verificó con la prueba ANOVA de un factor. Se encontró diferencia estadísticamente significativa en el índice total de las frases Adecuadas ( $p=.003, F=5.871, \mathrm{df}=2,294$ ), el efecto de esta diferencia es pequeño $(f=.19)$, con potencia estadística aceptable $(86 \%)$. Solamente en los índices de dos ítems se encontraron diferencias en función del grado de estudios, lo que se verificó con una prueba post hoc con el método Bonferroni. En la tabla 10 se presentan los resultados.

Tabla 10

Ítems con diferencia estadísticamente significativa en función del grado de estudios en algún índice, parámetros de la prueba ANOVA, tamaño del efecto (f) y potencia estadística

\begin{tabular}{l|l|l|c|c|c|c|c}
\hline Ítem & $\begin{array}{l}\text { Tipo de } \\
\text { índice }\end{array}$ & $\begin{array}{l}\text { Grupo con } \\
\text { mayor promedio }\end{array}$ & F & Df & p & f & $\begin{array}{l}\text { Potencia } \\
\text { estadística }\end{array}$ \\
\hline 40511 Bienestar económico & Ponderado & Posgrado & 6.070 & 2,294 & .003 & .19 & .87 \\
\hline 60411 Capacidades de científicos & Ponderado & Universitario & 3.966 & 2,294 & .020 & .28 & .99 \\
\hline \hline
\end{tabular}

Fuente: elaboración propia. ${ }^{*} p \leq .05$

Esta variable aportó diferencias en dos de los 19 ítems aplicados, lo que sugiere que la formación profesional tiene escasa influencia en las concepciones de NdC. La evidencia empírica apunta a que los programas educativos no promueven una visión adecuada de $\mathrm{NdC}$, pues se observa que ningún ítem de la dimensión Naturaleza del conocimiento científico muestra diferencia en sus índices, lo que coincide con lo reportado por María Mercedes Callejas-Restrepo y Édgar Alberto Mendoza-Parada (2011).

Asignatura que imparten. Se clasificó a los profesores de acuerdo con la principal asignatura que imparten como se observa en la tabla 11. Para determinar si los profesores de ciencias muestran concepciones más informadas de NdC, se aplicó la prueba ANOVA de un factor y no se encontraron diferencias estadísticamente significativas en ninguno de los índices globales. Tampoco se encontraron diferencias en los índices de ningún 
ítem, pues las que se detectaron tenían un efecto muy pequeño y una potencia estadística baja (Cárdenas-Castro \& Arancibia-Martini, 2014; Faul, Erdfelder, Lang \& Buchner, 2007). Este resultado sugiere que la formación científica que recibieron los participantes no aportó elementos para desarrollar concepciones más informadas de NdC, de modo que estas son similares a las de sus pares que imparten asignaturas del área de humanidades. El resultado coincide con lo reportado por Néstor Cardoso-Erlam y Edna Eliana Morales-Olivares (2011), quienes mencionan que esto puede ocurrir por el uso de modelos de enseñanza de las ciencias expositivos y carentes de reflexión epistemológica.

Tabla 11

Distribución de los profesores según la principal asignatura que imparten

\begin{tabular}{l|c|l|c}
\hline $\begin{array}{l}\text { Asignatura } \\
\text { que imparten }\end{array}$ & Frecuencia & $\begin{array}{l}\text { Asignatura } \\
\text { que imparten }\end{array}$ & Frecuencia \\
\hline Administrativo & 9 & Biología & 84 \\
\hline Humanidades & 17 & Física & 64 \\
\hline Matemáticas & 7 & Química & 84 \\
\hline Tecnología & 12 & Telesecundaria & 20 \\
\hline \hline
\end{tabular}

Fuente: elaboración propia. $n=297$

\section{Discusión de resultados y conclusiones}

Los resultados evidencian que las concepciones de NdC de los docentes evaluados son poco satisfactorias. La distribución de las puntuaciones medias de cada frase para toda la muestra se extienden desde un valor máximo de +.83 (en la frase Adecuada A60511G) hasta un valor mínimo de -.80 (en la frase Plausible P60511C). Las frases Adecuadas contribuyeron de forma positiva al índice global ponderado, mientras que las frases Ingenuas y Plausibles lo afectaron en sentido contrario. El índice global ponderado es negativo y próximo a cero $(\bar{x}=-.024, D E=.103)$, lo que coincide con el lugar secundario que se ha otorgado a la ciencia en la educación básica y que contribuye a preservar concepciones ingenuas sobre la ciencia (Flores-Camacho, 2012). Estas concepciones ingenuas prevalecen, ya que la NdC es un aspecto descuidado no solo en la educación secundaria, sino también en la normal y en la superior (Flores-Camacho, Gallegos-Cázares \& Reyes-Cárdenas, 2007). Los resultados apuntan a una deficiente formación en conocimiento pedagógico de contenido de $\mathrm{NdC}$ lo que invita a la instrucción explícita de estos contenidos en la formación docente inicial y continua (Lin, Lieu, Chen, Huang \& Chang, 2012).

El diagnóstico de las concepciones de $\mathrm{NdC}$ no tiene como finalidad clasificar personas de acuerdo a su grado de adecuación, sino describir y comparar posiciones complejas sobre estos temas (Vázquez-Alonso, Manassero-Mas \& Acevedo-Díaz, 2005) para proponer la intervención didáctica pertinente que ayude a los profesores a desarrollar el conocimiento pedagógico de contenido sobre NdC. En particular, la alta coincidencia con los expertos en las frases Adecuadas permite proponerlas como punto de partida que debe ser reforzado y actuar como soporte para mejorar los aspectos débiles o que causan confusión como es el caso de las frases Plausibles (Vázquez-Alonso, Acevedo-Díaz, Manassero-Mas \& AcevedoRomero, 2006). 
Las concepciones ingenuas de $\mathrm{NdC}$ que manifiestan los profesores evaluados apuntan al positivismo lógico, lo que coincide con investigaciones previas realizadas con profesores de secundaria (CarvajalCantillo \& Gómez-Vallarta, 2002; Flores-Camacho, Gallegos-Cázares, Bonilla-Pedroza, López \& García, 2007; Flores-Camacho, Gallegos-Cázares \& ReyesCárdenas, 2007), pues concuerdan en la visión de la ciencia como un conjunto de conocimientos que se van acumulando, desconocen la forma en que trabaja la ciencia y las características de los científicos; además de creer en un método científico universal e infalible, prevalece la visión de tecnología como ciencia aplicada y no tienen claridad sobre la relación entre ciencia, tecnología y sociedad. La persistencia del positivismo lógico puede ocurrir por la falta de oportunidades dentro de la formación docente para desarrollar actividades experimentales que les permitan comprender el proceso de la ciencia (Flores-Camacho, 2012).

Los profesores de secundaria mostraron concepciones de $\mathrm{NdC}$ mucho menos informadas que los profesores de educación media superior (Carvajal-Cantillo \& Gómez-Vallarta, 2002; Garritz, Rueda \& Robles, 2011; Rebollo-León, 2008) y superior (García-Ruiz, Calixto-Flores \& Cid del Prado, 2011; Garritz, Rueda \& Robles, 2011). Esto puede ocurrir porque en ese nivel educativo la actividad experimental es todavía más escasa, además de que normalmente no se incluye en los procesos de evaluación docente, lo que disminuye el interés por conocer la utilidad de los experimentos y su abordaje desde una mirada contemporánea (Flores-Camacho, 2012). Este resultado sugiere que los profesores tendrán dificultades para contribuir a la adecuada construcción de conocimiento en temas de NdC de sus estudiantes y por consiguiente no podrán desarrollar la competencia científica que requiere conocimientos de la ciencia y sobre la ciencia pues ellos mismos tienen dudas sobre cómo se genera el conocimiento científico.

Las variables sociodemográficas (género, edad y región de procedencia) aportaron más diferencias en conjunto que las variables de estatus profesional lo que sugiere que las condiciones objetivas de existencia que incluyen tanto las características del participante (sexo, edad, etc.) como las del entorno (físico, económico, social, etc.) (Giroux \& Tremblay, 2004), tienen mayor efecto en la concepción de $\mathrm{NdC}$; esto podría deberse a que las concepciones de $\mathrm{NdC}$ de los profesores se originan principalmente en su entorno social y cultural, influenciado particularmente por los medios de comunicación masiva (Flores-Camacho, Gallegos-Cázares \& Reyes-Cárdenas, 2007). Se detectaron mínimas diferencias estadísticamente significativas entre la concepción de NdC de hombres y mujeres, el efecto de esas diferencias es pequeño. En la mayoría de los casos, los hombres mostraron mejor puntuación, lo que contradice los resultados de una investigación previa (García-Ruiz, Calixto-Flores \& Cid del Prado, 2011). Jorge Luis Rebollo-León (2008) también detectó algunas diferencias en las concepciones de $\mathrm{NdC}$ entre hombres y mujeres, pero Nihal Dogan y Fouad Abd-ElKhalick (2008) sostienen que la visión de NdC no está relacionada con el género. En los casos en que hubo diferencias, la opinión de las mujeres estuvo más alejada de la visión de los expertos que las de los hombres por lo que se sugiere brindar más oportunidades de reflexión pedagógica sobre las concepciones de $\mathrm{NdC}$ a las docentes.

Al comparar por edad también se encontraron diferencias en las concepciones de $\mathrm{NdC}$, el efecto de las diferencias es pequeño; aunque la edad del docente y sus años de experiencia no necesariamente están relacionados, estudios previos han mostrado que los profesores con mayor experiencia tienden a exhibir visiones más ingenuas sobre la NdC (Rebollo-León, 2008; Tsai, 2002). Puede resultar útil orientar los cursos de formación considerando las concepciones de cada grupo etario. Por otro lado, este resultado puede ser positivo, aunque se tendría que demostrar. Si los profesores jóvenes tienen una mejor concepción de la $\mathrm{NdC}$, esto puede ser causado por un cambio en la educación a través de las generaciones. Para establecer si este es el motivo, se tendría que hacer un estudio longitudinal y ver cómo cambian las concepciones del profesor con la edad.

Finalmente, la región de procedencia también aportó diferencias significativas en las concepciones de $\mathrm{NdC}$, lo que coincide con lo reportado por Nihal Dogan y Fouad Abd-El-Khalick (2008), el efecto de las diferencias es pequeño, con excepción de la encontrada en el índice ponderado del ítem 60411 Capacidades de los científicos, que es mediano. Esto puede deberse al entorno social y cultural propio de cada región (Flores-Camacho, Gallegos-Cázares \& Reyes-Cárdenas, 2007), por lo que se sugiere considerar el contexto en el diseño de los cursos de formación docente, sumado a que fueron las variables sociodemográficas las que aportaron la mayor cantidad de diferencias en este estudio.

Solo la variable nivel de estudios, de las variables de estatus profesional, aportó diferencias cuyo efecto fue pequeño, por lo que se puede mencionar que los estudios profesionales no contribuyen a mejorar la concepción de NdC. Otros estudios no han encontrado evidencia suficiente de que los estudios universitarios para profesores modifiquen las concepciones de NdC (Callejas-Restrepo \& Mendoza-Parada, 2011); en el mismo sentido se encuentra que las concepciones de $\mathrm{NdC}$ de estudiantes de ciencias e ingenierías que inician y acaban sus estudios universitarios son más 
similares que diferentes, por lo que es necesario que la $\mathrm{NdC}$ no se considere un subproducto del aprendizaje de contenidos científicos e ingenieriles, sino que se aborde de forma explícita y con actividades metacognitivas de reflexión (Bennássar-Roig, García-Carmona, Vázquez-Alonso, Manassero-Mas \& Montesano de Talavera, 2011). Se recomienda entonces, integrar de manera explícita en los cursos de formación de profesores aspectos de $\mathrm{NdC}$.

Por último, al comparar por la asignatura que imparten, no se encontraron diferencias, por lo que puede mencionarse que los profesores de ciencias no profundizan en la reflexión de contenidos de NdC y sería conveniente proveerlos de guías educativas que los ayuden a mejorar tanto su comprensión de NdC como la enseñanza de estos contenidos (Lin, Lieu, Chen, Huang \& Chang, 2012). Se sugiere incorporar modelos didácticos de aprendizaje de la ciencia centrados en el estudiante mediante estrategias de aprendizaje activo, pues estos favorecen la comprensión de NdC al simular el trabajo científico, lo que favorece también la argumentación y reflexión epistemológica (Campos, Silva, Tecpan \& Zavala, 2016)

En general, las concepciones de $\mathrm{NdC}$ son ingenuas y se alejan de la visión de los expertos, por lo que los profesores no están en condiciones de apoyar a sus alumnos a desarrollar la competencia científica. Los índices detectados ponen de manifiesto que tanto la formación normal como la universitaria provocan cambios mínimos en las concepciones de $\mathrm{NdC}$ de los docentes, lo que coincide con lo reportado por otros autores (Callejas-Restrepo \& Mendoza-Parada, 2011; García-Ruiz, Calixto-Flores \& Cid del Prado, 2011; Garritz, Rueda \& Robles, 2011; Rebollo-León, 2008). Este resultado sugiere que los profesores continúan transmitiendo una imagen deformada del trabajo científico (Mosquera-Suárez, 2011). Es necesario desarrollar el conocimiento pedagógico de contenido de la NdC de los profesores de secundaria e incluirlo en los cursos de actualización docente mediante enseñanza explícita y con actividades metacognitivas de reflexión.

Si bien la muestra utilizada en este estudio es amplia, se reconoce que es limitada y de participación voluntaria. Adicionalmente, la encuesta se aplicó en formato electrónico por lo que es conveniente ampliar la muestra y emplear el formato presencial para verificar la consistencia en las respuestas. Por otra parte, de acuerdo con los autores del instrumento, este puede ser utilizado de manera cualitativa, aspecto que no se aplicó en este estudio pero que para futuras investigaciones podría emplearse para profundizar en las respuestas con mayor discrepancia con la visión de los expertos.

Los resultados del estudio permiten formular las siguientes conclusiones:

1. Las concepciones de $\mathrm{NdC}$ de los profesores de secundaria son ingenuas, de acuerdo con la clasificación propuesta por José Antonio Acevedo-Díaz, Ángel Vázquez-Alonso, María Antonia ManasseroMas y Pilar Acevedo-Romero (2007) y se alejan de la visión de los expertos, principalmente en ítems relacionados con la manera de aproximarse a las investigaciones, rubro en que se obtuvo una de las puntuaciones más bajas en el índice global ponderado. Este resultado es significativo pues podría explicar los bajos resultados de México en las evaluaciones de PISA. En los cursos en secundaria se enfatiza el contenido, que se transmite mediante modelos de enseñanza expositivos, sin incorporar la reflexión sobre la construcción de estos contenidos y de la importancia de la indagación en su construcción que pueden ser elementos clave para el desarrollo de la competencia científica. Se coincide con lo reportado por Andoni Garritz, Cristina Rueda y César Robles (2011), quienes sugieren que las concepciones ingenuas son resultado de la falta de formación en 
aspectos de NdC. Es necesario que en las clases de ciencias actuales se destine el tiempo de exposición de hechos y contenidos a la reflexión e indagación de manera rutinaria de tal forma que se potencie el desarrollo de la competencia científica, para ello es preciso mejorar la actualización y formación docente en $\mathrm{NdC}$.

2. Las concepciones de $\mathrm{NdC}$ de los profesores de secundaria son casi tan ingenuas como las de los estudiantes; esto coincide con hallazgos en México en niveles educativos superiores (CallejasRestrepo \& Mendoza-Parada, 2011; García-Ruiz, Calixto-Flores \& Cid del Prado, 2011; Garritz, Rueda \& Robles, 2011; Rebollo-León, 2008) y de acuerdo con los autores, puede encontrar su origen en la falta de formación explícita, lo que dificulta que los profesores logren desarrollar la competencia científica en sus alumnos, ya que un componente esencial de esta competencia es la comprensión de la NdC. Un problema que se enfrenta en México, y probablemente en otros países de Latinoamérica, es la necesidad de reformar las clases de ciencia de secundaria para enfatizar no solo en el conocimiento, sino también en la construcción del conocimiento científico mediante la reflexión e indagación. Sin embargo, la preparación de los profesores al respecto es débil. Es necesario establecer programas de formación docente con un fuerte componente de desarrollo de la competencia científica y no solo de los profesores de secundaria, sino también de los profesores de educación básica.

3. Las variables sociodemográficas mostraron mayor incidencia en las concepciones de $\mathrm{NdC}$ que las variables de estatus profesional, lo que sugiere que el conocimiento de $\mathrm{NdC}$ se ha obtenido mediante educación informal, quizá influenciada por la cultura de la región. Si bien Fernando Flores-Camacho, Leticia Gallegos-Cázares, María Xóchitl Bonilla-Pedroza, Luz Iris López y Beatriz García (2007) proponen que la tendencia al positivismo lógico, que no se considera una concepción contemporánea de $\mathrm{NdC}$, puede tener su origen en los procesos de formación de los docentes, también reconocen que está influenciada por elementos informales como la divulgación científica, el sentido común y otros aspectos contextuales de modo que se propone incorporar aspectos culturales y contextuales en los programas de formación docente para prevenir que esta visión poco adecuada de $\mathrm{NdC}$ se siga transmitiendo a otras generaciones de estudiantes.

4. Las variables de estatus profesional prácticamente no modifican las concepciones de $\mathrm{NdC}$ de los profesores de secundaria, lo que refuerza que la formación disciplinar, independientemente de la profesión de origen, no ha contribuido a modificar las concepciones de NdC. Los resultados de Néstor Cardoso-Erlam y Edna Eliana Morales-Olivares (2011) y de Mayra García-Ruiz, Raúl Calixto-Flores y Alejandro Cid del Prado (2011) destacan que estudiantes y profesores de ciencias y humanidades sostienen concepciones similares de $\mathrm{NdC}$, lo que implica que la formación científica no contribuye al desarrollo de conocimiento sobre la ciencia, aspecto que puede ocurrir por el tipo de estrategias didácticas empleadas, además de la falta de oportunidades para desarrollar trabajo similar al de los científicos. Se coincide con los autores en la urgente necesidad de revisar planes y programas de formación en ciencias que incorporen de manera explícita y concreta contenidos de $\mathrm{NdC}$. 
Con esto en mente, se propone lo siguiente:

1. A partir de las fortalezas detectadas en el diagnóstico, diseñar cursos de actualización profesional sobre NdC que incluyan metacognición y reflexión de la práctica docente. Estas recomendaciones se hacen a la formación de profesores en las diferentes regiones del país para tratar, por una parte, de mejorar las concepciones de los profesores con respecto a la NdC, pero por otro, homogeneizar estas concepciones en las diferentes regiones del país.

2. Considerar las diferencias asociadas a las variables sociodemográficas para diseñar cursos contextualizados de $\mathrm{NdC}$ que propicien mayor interés pues — como se evidenció en los resultados- estas variables son las que aportan mayores elementos de diferenciación en las concepciones de $\mathrm{NdC}$ de los profesores. No se propone que los cursos de actualización sean diametralmente diferentes, pues finalmente las concepciones de $\mathrm{NdC}$ de todos los profesores del país estuvieron alejadas de los expertos; sin embargo, es necesario tomar en cuenta la cultura regional donde pueda haber influencia. En este estudio no se establecieron diferencias entre profesores de comunidades urbanas y rurales pues no se tenían los datos, no obstante, este puede ser un factor a tomarse en cuenta.

3. Adoptar el enfoque explícito en la enseñanza de la NdC, en los planes de estudio de los distintos niveles educativos mediante el uso de la filosofía e historia de la ciencia, para reorientar las concepciones de $\mathrm{NdC}$ adquiridas en la formación profesional que pueden seguir encaminadas hacia el positivismo lógico. Proponemos que estas adaptaciones se hagan en los cursos de ciencias y no en cursos específicos de NdC. Los estudiantes (y los profesores) deben comprender que la ciencia y la naturaleza de la ciencia son intrínsecas.

4. Continuar con la formación profesional en competencia científica que, aparte de concepciones informadas de $\mathrm{NdC}$, incluye habilidades de razonamiento científico y actitudes hacia la ciencia. Es necesario establecer un plan de capacitación docente que mejore y refuerce no solo las concepciones de $\mathrm{NdC}$, sino que contribuya al desarrollo de estas competencias no adquiridas en la formación profesional.

5. Profundizar en la indagación de la relación de las concepciones de $\mathrm{NdC}$ y su efecto en la práctica docente. A mayor cantidad de docentes capaces de reflexionar sobre su propio entendimiento de las ciencias y su naturaleza, habrá mayor posibilidad de obtener resultados más contundentes de estudios similares al que se presenta. Nuestro trabajo en particular, va en este camino.

\section{Agradecimientos}

Los autores agradecen el apoyo de la Cátedra de Investigación e Innovación en la Educación de la Física del Tecnológico de Monterrey, Campus Monterrey.

\section{Sobre los autores}

Silvia Tecpan - Flores es ingeniera industrial del Instituto Politécnico Nacional, IPN. Doctora en innovación educativa y maestra en educación, Universidad Virtual del Tecnológico de Monterrey, Escuela de Humanidades y Educación. Profesora asistente del departamento de Física, Universidad de Santiago de Chile y profesora de cátedra en la Escuela de Humanidades y Educación, ITESM. 
Genaro Zavala - Enríquez es ingeniero físico industrial, Tecnológico de Monterrey. Doctor en ciencia y tecnología del estado sólido, Syracuse University. Dirige el Grupo de Investigación e Innovación en la Educación de la Física y trabaja con las siguientes líneas de investigación: entendimiento conceptual de estudiantes sobre temas de física, transferencia de entendimiento entre las diferentes áreas del conocimiento, uso de tecnologías en el aprendizaje, impacto del uso de ambientes de aprendizaje innovadores y desarrollo de herramientas de evaluación.

\section{Referencias}

Acevedo-Díaz, José Antonio; Vázquez-Alonso, Ángel; Acevedo-Romero, Pilar \& Manassero-Mas, María Antonia (2002). Sobre las actitudes y creencias CTS del profesorado de primaria, secundaria y universidad. Tarbiya. Revista de Investigación e Innovación Educativa, 30, 5-27. Disponible en: http://www.uam.es/servicios/apoyodocencia/ice/tar biya/pdf/revistas/Tarbiya030.pdf

Acevedo-Díaz, José Antonio; Vázquez-Alonso, Ángel; Manassero-Mas, María Antonia \& Acevedo-Romero, Pilar (2002). Persistencia de las actitudes y creencias CTS en la profesión docente. Revista Electrónica de Enseñanza de las Ciencias, 1 (1), 1-27. Disponible en: http://www. saum.uvigo.es/reec/volumenes/volumen1/Numero1/art1.pdf

Acevedo-Díaz, José Antonio; Vázquez-Alonso, Ángel; Manassero-Mas, María Antonia \& Acevedo-Romero, Pilar (2007). Consensos sobre la naturaleza de la ciencia: Fundamentos de una investigación empírica. Revista Eureka sobre Enseñanza y Divulgación de las Ciencias, 4 (1), 42-66. Disponible en: http://www.redalyc.org/articulo. oa?id $=92040104$

Arnett, Jeffrey Jensen (2008). Adolescencia y adultez emergente, un enfoque cultural. Ciudad de México: Pearson. Disponible en: http:// www.dandrosh.com.mx/books/Adolescencia\%20Y\%20Adultez $\% 20$ Emergente\%20Un\%20Enfoque\%20Cultural\%20-\%20Jeffrey $\% 20$ Jensen\%20Arnett.pdf

Barona-Ríos, César; Verjovsky, Janet Paul Wright de; Moreno-Ruiz, Marcela \& Lessard, Claude (2004). La concepción de la naturaleza de la ciencia (CNC) de un grupo de docentes inmersos en un programa de formación profesional en ciencias. Revista Electrónica de Investigación Educativa, 6 (2), 1-19. Disponible en: http://redie.uabc.mx/vol6no2/ contenido-barona.html

Bennássar-Roig, Antoni; García-Carmona, Antonio; Vázquez-Alonso, Ángel; Manassero, María Antonia \& Montesano de Talavera, Marisa (2011). ¿Aportan algo los estudios universitarios de grado a la comprensión de NdCyT? En Antoni Bennássar-Roig, Ángel VázquezAlonso, María Antonia Manassero-Mas \& Antonio García-Carmona (coords.). Ciencia, tecnología y sociedad en Iberoamérica: una evaluación de la comprensión de la naturaleza de ciencia y tecnología, 129-137. Documentos de Trabajo, 5. Madrid: Centro de Altos Estudios Universitarios de la Organización de Estados Iberoamericanos para la Educación, la Ciencia y la Cultura (OEI). Disponible en: http:// www.oei.es/historico/cienciayuniversidad/spip.php?article1862

Bennássar-Roig, Antoni; Vázquez-Alonso, Ángel; Manassero-Mas, María Antonia \& García-Carmona, Antonio (coords.) (2011). Ciencia, tecnología y sociedad en Iberoamérica: una evaluación de la comprensión de la naturaleza de ciencia y tecnología, 129-137. Documentos de Trabajo, 5. Madrid: Centro de Altos Estudios Universitarios de la Organización de Estados Iberoamericanos para la Educación, la 
Ciencia y la Cultura (OEI). Disponible en: http:// www.oei.es/historico/cienciayuniversidad/spip. php?article1862

Cakici, Yilmaz \& Bayir, Eylem (2012). Developing Children's Views of the Nature of Science through Role-Play. International Journal of Science Education, 34 (7), 1075-1091.

Callejas-Restrepo, María Mercedes \& Mendoza-Parada, Édgar Alberto (2011). Diferencias en la comprensión de NdCyT entre profesores que inician la universidad y los que finalizan su grado. En Antoni Bennássar-Roig, Ángel Vázquez-Alonso, María Antonia Manassero-Mas \& Antonio García-Carmona (coords.). Ciencia, tecnología y sociedad en Iberoamérica: una evaluación de la comprensión de la naturaleza de ciencia y tecnología, 89-100. Documentos de Trabajo, 5. Madrid: Centro de Altos Estudios Universitarios de la Organización de Estados Iberoamericanos para la Educación, la Ciencia y la Cultura (OEI). Disponible en: http://www.oei.es/historico/cien ciayuniversidad/spip.php?article1862

Campos, Esmeralda; Silva, Leonor; Tecpan, Silvia \& Zavala, Genaro (2016). Argumentation during Active Learning Strategies in a SCALE-UP Environment. En Dyan L. Jones, Lin Ding \& Adrienne L. Traxler (eds.). Physics Education Research Conference, 64-67. Sacramento, California: American Association of Physics Teachers. doi:10.1119/ perc.2016.pr.011. Disponible en: http://www. per-central.org/items/detail.cfm?ID=14194

Candela, Antonia (2006). Comentarios a los programas de ciencias I, II y III en el marco de la RES [Reforma a la Educación Secundaria]. Revista Mexicana de Investigación Educativa, 11 (31), 1451-1462. Disponible en: http://www.redalyc. org/html/140/14003116/

Cañas, Ana; Martín-Díaz, María Jesús \& Nieda, Juana (2007). Competencia en el conocimiento y la interacción con el mundo físico. La competencia científica. Madrid: Alianza.

Cárdenas-Castro, Manuel \& Arancibia-Martini, Héctor (2014). Potencia estadística y cálculo del tamaño del efecto en $\mathrm{G}^{*}$ Power: complementos a las pruebas de significación estadística y su aplicación en psicología//Statistical Power and Effect Size Calculating in $\mathrm{G}^{*}$ Power: Complementary Analysis of Statistical Significance Testing and its Application in Psychology. Salud \& Sociedad, 5 (2), 210-224. Disponible en: https://dialnet.uni rioja.es/descarga/articulo/4945415.pdf

Cardoso-Erlam, Néstor \& Morales-Olivares, Edna Eliana (2011). ¿Son diferentes las actitudes hacia la NdCyT y sociedad por parte de los estudiantes y profesores de ciencias y de humanidades? Un estudio en seis países iberoamericanos. En Antoni Bennássar-Roig, Ángel Vázquez-Alonso, María Antonia Manassero-Mas \& Antonio García-Carmona (coords.). Ciencia, tecnología y sociedad en Iberoamérica: una evaluación de la comprensión de la naturaleza de ciencia y tecnología, 151164. Documentos de Trabajo, 5. Madrid: Centro de Altos Estudios Universitarios de la Organización de Estados Iberoamericanos para la Educación, la Ciencia y la Cultura (OEI). Disponible en: http://www.oei.es/historico/cienciayuniversidad/ spip.php?article1862

Carvajal-Cantillo, Enna \& Gómez-Vallarta, María del Rocío (2002). Concepciones y representaciones de los maestros de secundaria y bachillerato sobre la naturaleza, el aprendizaje y la enseñanza de las ciencias. Revista Mexicana de Investigación Educativa, 7 (16), 577-602.

Chin, Chi-Chin (2005). First-year Pre-service Teachers in Taiwan. Do they Enter the Teacher Program with Satisfactory Scientific Literacy and Attitudes toward Science? International Journal of Science Education, 27 (13), 1549-1570. doi:10.1080/09585190500186401. Disponible en: http://www.ntcu.edu.tw/chin/file/30.pdf

Dogan, Nihal \& Abd-El-Khalick, Fouad (2008). Turkish Grade 10 Students' and Science Teachers' Conceptions of Nature of Science: A National Study. Journal of Research in Science Teaching, 45 (10), 1083-1112. doi:10.1002/tea.20243

Faul, Franz; Erdfelder, Edgar; Lang, Albert-Georg \& Buchner, Axel (2007). G*Power 3: A Flexible Statistical Power Analysis Program for the Social, Behavioral, and Biomedical Sciences. Behavior Research Methods, 39 (2), 175-191. Doi: 10.3758/BF03193146

Flores-Camacho, Fernando (2012). Conocimientos, concepciones y formación de los profesores. En Fernando Flores-Camacho (coord.). La enseñanza de la ciencia en la educación básica en México, 113-128. Ciudad de México: Instituto Nacional para la Evaluación de la Educación, INEE. Disponible en: http://publicaciones.inee.edu.mx/ buscadorPub/P1/C/227/P1C227.pdf

Flores-Camacho, Fernando; Gallegos-Cázares, Leticia; Bonilla-Pedroza, María Xóchitl; López, Luz Iris \& García, Beatriz (2007). Concepciones sobre la naturaleza de la ciencia de los profesores de biología del nivel secundario. Revista Mexicana de Investigación Educativa, 12 (32), 359380. Disponible en: http://www.redalyc.org/ pdf/140/14003217.pdf

Flores-Camacho, Fernando; Gallegos-Cázares, Leticia \& Reyes-Cárdenas, Flor (2007). Perfiles y orígenes de las concepciones de ciencia de los 
profesores mexicanos de química. Perfiles Educativos, 24 (116), 6084. Disponible en: http://www.redalyc.org/articulo.oa?id=13211604 Flores-Camacho, Fernando; López-Mota, Ángel; Gallegos-Cázares, Leticia \& Barojas, Jorge (2000). Transforming Science and Learning Concepts of Physics Teachers. International Journal of Science Education, 22 (2), 197-208. doi:10.1080/095006900289958

García-Ruiz, Mayra; Calixto-Flores, Raúl \& Cid del Prado, Alejandro (2011). Creencias sobre la NdCyT: Una comparación entre estudiantes universitarios de ciencias y de humanidades. En Antoni Bennássar-Roig, Ángel Vázquez-Alonso, María Antonia Manassero-Mas \& Antonio García-Carmona (coords.). Ciencia, tecnología y sociedad en Iberoamérica: una evaluación de la comprensión de la naturaleza de ciencia y tecnología, 179-192. Documentos de Trabajo, 5. Madrid: Centro de Altos Estudios Universitarios de la Organización de Estados Iberoamericanos para la Educación, la Ciencia y la Cultura (OEI). Disponible en: http://www.oei.es/historico/cienciayuniversidad/spip. php?article1862

Garritz, Andoni; Rueda, Cristina \& Robles, César (2011). Opiniones de profesores y estudiantes mexicanos del bachillerato y la universidad públicos sobre la NdCyT: una pobreza alarmante. En Antoni Bennássar-Roig, Ángel Vázquez-Alonso, María Antonia Manassero-Mas \& Antonio García-Carmona (coords.). Ciencia, tecnología y sociedad en Iberoamérica: una evaluación de la comprensión de la naturaleza de ciencia y tecnología, 115-125. Documentos de Trabajo, 5. Madrid: Centro de Altos Estudios Universitarios de la Organización de Estados Iberoamericanos para la Educación, la Ciencia y la Cultura (OEI). Disponible en: http://www.oei.es/historico/cienciayuniversidad/spip. php?article1862

Giroux, Sylvain \& Tremblay, Ginette (2004). Metodología de las ciencias humanas. Ciudad de México: Fondo de Cultura Económica, FCE.

Lederman, Norman G. (2007). Nature of Science: Past, Present and Future. En Sandra K. Abell \& Norman G. Lederman (eds.). Handbook of Research on Science Education, 831-880. Mahwah, New Jersey: Lawrence Erlbaum Associates. Disponible en: http://www.csss-scien ce.org/downloads/NOS_Lederman_2006.pdf

Lin, Shu Fen; Lieu, Sang Chong; Chen, Sufen; Huang, Mao Tsai \& Chang, Wen Hua (2012). Affording Explicit-Reflective Science Teaching by Using an Educative Teachers' Guide. International Journal of Science Education, 34 (7), 999-1026. doi:10.1080/09500693.2012.661484

López y Mota, Ángel Daniel \& León-Trueba, Ana Isabel (2003). Currículo como estructura y proceso. En Ángel Daniel López y Mota (coord.). La investigación educativa en México 1992-2002: saberes científicos, humanísticos y tecnológicos, 369-456. Ciudad de México: Consejo Mexicano de Investigación Educativa, COMIE. Disponible en: http:// www.comie.org.mx/doc/portal/publicaciones/ec2002/ec2002_v07_ t1.pdf

López y Mota, Ángel Daniel; Rodríguez-Pineda, Diana Patricia \& Bonilla-Pedroza, María Xóchitl (2004). ¿Cambian los cursos de actualización las representaciones de la ciencia y la práctica docente? Revista Mexicana de Investigación Educativa, 9 (22), 699-719. Disponible en: http:// www.oei.es/docentes/articulos/cambian_cursos_actualizacion_re presentaciones_practica_docente.pdf

Manassero-Mas, María Antonia \& Vázquez-Alonso, Ángel (2001). Actitudes y creencias de los estudiantes relacionadas con CTS. En Pedro Membiela-Iglesia (ed.). Enseñanza de las ciencias desde la perspectiva 
ciencia-tecnología-sociedad. Formación científica para la ciudadanía, 149-162. Madrid: Narcea.

Manassero-Mas, María Antonia; Vázquez-Alonso, Ángel \& AcevedoDíaz, José Antonio (2001). Cuestionario de opiniones sobre ciencia, tecnología y sociedad, COCTS [Manual]. Palma de Mallorca, España. Disponible en: https://www.researchgate.net/profile/Jose_AcevedoDiaz/publication/261070841_Cuestionario_de_Opiniones_sobre_ Ciencia_Tecnologia_y_Sociedad_COCTS_-_Manual_Modelos_de_ respuesta_y_puntuacion_Views_on_Science-Technology-Society_ Questionnaire___Guide_for_response_and_scoring_models/ links/0a85e533293324e0a8000000.pdf?origin=publication_detail

Mosquera-Suárez, Carlos Javier (2011). La investigación sobre la formación de profesores desde la perspectiva del cambio didáctico. magis, Revista Internacional de Investigación en Educación, 3 (6), 265-282. Disponible en: http://revistas.javeriana.edu.co/index.php/MAGIS/article/ view/3541/2645

Organisation for Economic Cooperation and Development, OECD (2010). PISA 2009 Results: Executive Summary. Disponible en: http://www. oecd.org/pisa/pisaproducts/46619703.pdf

Organización de las Naciones Unidas para la Educación la Ciencia y la Cultura, UNESCO (2010). Informe de la UNESCO sobre la ciencia 2010. El estado actual de la ciencia en el mundo. Susan Schneegans (ed.). París: Ediciones UNESCO. Disponible en: http://unesdoc.unesco.org/ images/0018/001898/189883s.pdf

Organización para la Cooperación y el Desarrollo Económicos, OCDE (2008). Informe PISA 2006. Competencias científicas para el mundo del mañana. Madrid: Santillana. Disponible en: http://www.oecd-ili brary.org/education/informe-pisa-2006_9789264066205-es, http:// dx.doi.org/10.1787/9789264040014-en

Oviedo-Acevedo, Heidi Celina \& Campo-Arias, Adalberto (2005). Aproximación al uso del coeficiente alfa de Cronbach. Revista Colombiana de Psiquiatría, 34 (4), 572-580. Disponible en: https://goo.gl/IKqaqi, http://www.redalyc.org/pdf/806/80634409.pdf

Pozo-Municio, Juan Ignacio \& Gómez-Crespo, Miguel Ángel (1998). Aprender y enseñar ciencia. Madrid: Morata.

Pro Bueno, Antonio José de (2003). La construcción del conocimiento científico y los contenidos de ciencias. En María Pilar Jiménez-Aleixandre (ed.). Enseñar ciencias, 33-54. Barcelona: Graó.

Rebollo-León, Jorge Luis (2008). Preconcepciones de ciencia y tecnología en los profesores de bachillerato: un estudio empírico en el estado de Guanajuato. Revista Electrónica Iberoamericana sobre Calidad, Eficacia y Cambio en Educación. REICE, 6 (1), 119-133. Disponible en: http://www.rinace.net/arts/vol6num1/art7.pdf

Sheskin, David J. (2007). Handbook of Parametric and Nonparametric Statistical Procedures. New York: Chapman \& Hall.

Talanquer, Vicente (2000). El movimiento CTS [Ciencia-Tecnología-Sociedad] en México, ¿vencedor o vencido? Educación Química, 11 (4), 381-386. Disponible en: http://www.bmb.arizona.edu/tpp/Ed Quim00.pdf

Tsai, Chin-Chung (2002). Nested Epistemologies: Science Teachers' Beliefs of Teaching, Learning and Science. International Journal of Science Education, 24 (8), 771-783. doi:10.1080/0950069011 0049132. Disponible en: https://ir.nctu.edu.tw/bitstream/11536/ 14482/1/000177250000001.pdf 
Valdés-Cuervo, Ángel Alberto; Arreola-Olivarría, Claudia Gabriela; Angulo-Armenta, Joel; Carlos-Martínez, Ernesto Alonso \& García-López, Ramona Imelda (2011). Actitudes de docentes de educación básica hacia las TIC. magis, Revista Internacional de Investigación en Educación, 3 (6), 379-392. Disponible en: http://revistas.javeriana.edu.co/ index.php/MAGIS/article/view/3546/2650

Vázquez-Alonso, Ángel; Acevedo-Díaz, José Antonio; Manassero-Mas, María Antonia \& Acevedo-Romero, Pilar (2006). Actitudes del alumnado sobre ciencia, tecnología y sociedad, evaluadas con un modelo de respuesta múltiple. Revista Electrónica de Investigación Educativa, 8 (2), 1-37. Disponible en: http://redie.uabc.mx/vol8no2/contenidovazquez2.html

Vázquez-Alonso, Ángel; Acevedo-Díaz, José Antonio \& Manassero-Mas, María Antonia (2005). Más allá de la enseñanza de las ciencias para científicos : hacia una educación científica humanística. Revista Electrónica de Enseñanza de las Ciencias, 4 (2). Disponible en: http://reec. uvigo.es/volumenes/volumen4/ART5_Vol4_N2.pdf

Vázquez-Alonso, Ángel; Manassero-Mas, María Antonia \& Acevedo-Díaz, José Antonio (2005). Análisis cuantitativo de ítems complejos de opción múltiple en ciencia, tecnología y sociedad: escalamiento de ítems. Revista Electrónica de Investigación Educativa, 7 (1), 1-31. Disponible en: http://www.redalyc.org/articulo.oa?id=15507105, http://redie. uabc.mx/index.php/redie/article/view/116.org/pdf/155/15507105.pdf

Vázquez-Alonso, Ángel; Manassero-Mas, María Antonia; Acevedo-Díaz, José Antonio \& Acevedo-Romero, Pilar (2007). Consensos sobre la naturaleza de la Ciencia: la comunidad tecnocientífica. Revista Electrónica de Enseñanza de las Ciencias, 6 (2), 331-363. Disponible en: http://reec.uvigo.es/volumenes/volumen6/ART7_Vol6_N2.pdf

Vázquez-Alonso, Ángel; Manassero-Mas, María Antonia \& Talavera, Marisa (2010). Actitudes y creencias sobre naturaleza de la ciencia y la tecnología en una muestra representativa de jóvenes estudiantes. Revista Electrónica de Enseñanza de las Ciencias, 9 (2), 333-352. Disponible en: http://reec.uvigo.es/volumenes/volumen9/ART3_Vol9_N2.pdf

Verjovsky, Janet Paul Wright de \& Waldegg, Guillermina (2005). Analyzing Beliefs and Practices of a Mexican High School Biology Teacher. Journal of Research in Science Teaching, 42 (4), 465-491. doi:10.1002/ tea.20059

Vogt, W. Paul (2007). Quantitative Research Methods for Professionals. Boston, Massachusetts: Pearson. 